Anna Czekaj

Université de Silésie, Katowice

Pologne

(D) https://orcid.org/0000-0003-0606-5393

\section{Classement de métonymies et traduction automatique}

\title{
Classification of metonymy and automatic translation
}

\begin{abstract}
The paper is a continuation of the author's previous work regarding the legitimacy of metonymy classification for the needs of automatic translation. The author attempts to solve the problem presented in the paper entitled Classification of metonymy and its usefulness in automatic translation and proposes her classification of metonymic expressions, which could be a helpful tool in computer translation.
\end{abstract}

\section{Keywords}

Metonymy, automatic translation, object-oriented approach, lexicographic description, object class, attributes, operators, frame, classification

Le présent article se situe dans le cadre de la description lexicographique pour les besoins de la traduction automatique, proposée par l'approche orientée objets de W. B a ny ś (2002a, 2002b) et constitue la continuation de notre article précédent "Classement de métonymies et son utilité dans la traduction automatique » (A. C z e k a j, 2019) consacré à l'analyse de différentes typologies de métonymies, qui, dans la littérature sur le sujet se présentent en abondance (cf. p. ex. G. L a k off, M. Johnson, 1980 [1988] ; H. Morier, 1961 [1989]; D. Stallard, 1993 ; Z. Kövecses, G. Radden, 1998 ; A. Blank, 1999 ; Y. Peirsman, D. Geeraerts, 2006 ; P.J. Arnaud, 2009). Parmi les classifications présentées, celle qui nous a paru particulièrement intéressante du point de vue de l'objectif visé, était la proposition de T. Mas s o u s s i (2007, 2008, 2009), qui dans sa typologie des métonymies, élaborée pour les besoins de son utilisation 
ultérieure dans le traitement automatique, s'appuie, comme nous, sur la méthodologie des classes d'objets, néanmoins telle qu'elle a été présentée par G. Gros s (1994a, 1994b, 1995, 2008).

Rappelons donc brièvement que la classe d'objets, qui constitue l'élément central aussi bien de la conception de G. Gross que de celle de W. Banyś est une classe sémantique " construite à partir des prédicats (répartis en attributs et opérateurs) qui permettent de sélectionner de façon appropriée les unités qui la composent »(A. Grigowicz, 2007 : 231 ; cf. G. Gros s, 1994a, 1994b, 1995 ; W. B a nyś, 2002a, 2002b). Par conséquent, les éléments de la même classe d'objets sont caractérisés par les mêmes ensembles d'attributs et opérateurs. On voit ainsi que la définition de l'objet dans les deux conceptions a le caractère opérationnel, car on ne peut la connaître que grâce à l'analyse de toutes les opérations que cet objet " peut effectuer ou qui peuvent être effectuées sur lui » (W. B a n y ś, 2002a : 17). En général, toutes ces informations sont à trouver dans l'entourage immédiat de l'objet analysé en tant qu'adjectifs et verbes qui l'accompagnent, permettant de préciser le sens dans lequel il est employé dans une situation (phrase) concrète. Ainsi, l'importance du contexte dans la description des objets (des unités lexicales) témoigne que, primo - cette caractéristique est directement tributaire du comportement linguistique des objets, c'est-à-dire de « la façon dont la langue considère les entités extralinguistiques » (W. B a n y ś, 2002a : 17), secundo - les objets ne sont pas traités comme des entités isolées et leur comportement, et par conséquent la signification, émerge dans le contexte.

De ce point de vue, la description du lexique, en termes de classes d'objets, vise à rendre compte qu'en fonction de l'objet qui est traité et auquel on attribue différents prédicats, le fonctionnement d'une unité linguistique donnée peut changer à chaque fois (cf. G. Gross, 1994, 1995, 2008 ; A. Czek aj, 2014, 2019).

L'approche de ce type a été appliquée par T. Massoussi à la description des métonymies, qui, d'après l'auteur, résulteraient « d'un transfert de prédicats entre des unités lexicales »(T. Mas so ussi, 2008 : 29). Ce transfert est basé sur une correspondance lexicale et syntaxique entre des classes de départ (classes sources) et des classes d'arrivée (classes cibles). T. Massoussi ajoute encore, que si l'on aspire à une classification précise des unités lexicales en classes d'objets convenables, il faudrait considérer le transfert non pas dans le cadre des classes trop générales mais plutôt dans le cadre des classes plus précises. De cette façon, les classes d'objets permettraient de spécifier, par exemple, quels contenants empruntent les prédicats à quels contenus ou quels résultats sont générés par quelles actions. Une telle appropriation « qui décrit la dépendance entre des classes d'arguments et des classes de prédicats»(T. Mas sous si, 2008 : 31) peut être, selon l'auteur, très efficace non seulement pour créer les phrases métonymiques du type, p. ex. : 
(1) J'ai bu un verre (au lieu de J'ai bu un verre de vin)

(2) J'ai fini mon assiette (au lieu de J'ai fini une (mon) assiette de potage)

mais aussi pour éviter les constructions incorrectes telles que, p. ex. :

(3) ? J'ai plié ma valise (pour J'ai plié mes vêtements)

(4) ? J'ai mangé un sac (pour J'ai mangé des pommes de terre)

T. Massoussi justifie son point de vue par la constatation que c'est parce que «verre et potage sont des contenus appropriés respectivement à verre et à assiette qu'il y a la métonymie dans les phrases (1) et (2), ce qui n'est pas le cas des exemples (3) et (4) car vêtements n'est pas approprié à valise, ni pommes de terre à $s a c »$ (T. M a s o u s si, 2008 : 31). Il fait remarquer ainsi que la métonymie ne s'établit pas entre les catégories générales des noms des contenants et des noms des contenus, mais « entre les noms des [boissons] (vin), et les noms des [récipients] (verre) dans (5) » (T. Mas soussi, 2008 : 31), ce qui permettrait d'expliquer la possibilité de former d'autres métonymies comme p. ex. :

(5) Jean a sifflé deux verres.

(6) Il est en train de déguster une bouteille avec des camarades.

(7) On va prendre un pot tous ensemble dans une petite boîte, au Quartier latin.

ainsi que l'impossibilité d'emploi métonymique avec le verbe p. ex. regarder dans la phrase suivante :

(8) J'ai regardé un verre (pour J'ai regardé le vin)

étant donné que le verbe en question, n'est pas approprié à la classe des [boissons] et de ce fait, ne peut pas générer les constructions métonymiques. Or, même si le verbe regarder n'est certainement pas un opérateur typique pour la classe mentionnée, il n'est pas interdit à cette classe non plus. En effet, on pourrait trouver une bonne justification de l'emploi de la phrase citée ci-dessus, si l'on s'imaginait, par exemple, un alcoolique qui essaye de suivre un traitement de désintoxication mais qui dans une situation difficile et désespérante, se rend à un bar où $i l$ regarde des verres ne voyant que de l'alcool dedans, tellement il a du mal à se retenir de boire un coup (cf. A. C ze kaj, 2019).

De l'autre côté, la métonymie dans la phrase (1) J'ai bu un verre, ne doit pas forcément faire allusion au vin, ce récipient étant aussi bien approprié pour l'eau, le jus de fruits, le lait ou d'autres boissons.

De nombreux exemples de ce type, où la métonymie, considérée par T. Massoussi comme fautive car impossible du point de vue du transfert des prédicats, 
se manifestent pourtant dans la langue, ce que nous avons illustré de multiples contre-exemples correspondants (cf. A. C z ek aj, 2019).

Par conséquent, l'analyse de la typologie des métonymies présentée par T. Massoussi, dont le but était de rendre compte de la possibilité ou l'impossibilité du transfert des prédicats entre les classes « sources » et les classes « cibles » déterminées, nous a poussée à réfléchir sur le bien-fondé d'une classification si détaillée et rigoureuse pour les besoins de la traduction automatique. Nous nous sommes posé la question de savoir si une telle classification est vraiment indispensable et si elle facilite à la machine le choix de l'équivalent convenable dans la langue d'arrivée.

Étant donné que la qualité de la traduction automatique dépend directement de la qualité de la description des unités lexicales introduite dans la base des données de l'ordinateur, on pourrait croire, tout au moins à première vue, que plus cette description est détaillée plus il y a de chances que la traduction obtenue sera correcte. Ceci dit, il faudrait cependant ajouter que trop de précisions peut rendre la description trop rigide pour nombre d'expressions linguistiques qui échappent aux strictes règles auxquelles on voudrait les soumettre. Parmi ces expressions, on trouverait sans aucun doute des métonymies, surtout des métonymies vives « qui sont particulièrement susceptibles de désobéir aux règles rigides de la langue en tant que constructions généralement occasionnelles et imprévisibles » (A. Cze k aj, 2019 : 119 ; cf. M. L e c olle, 2001).

Ainsi, ayant reconnu le caractère inefficace et inutile de toute classification ferme et définitive des métonymies, étant donné que pour chaque typologie distinguée on pourrait toujours trouver des exemples opposés qui la mettent en question, nous voudrions proposer une approche plus tolérante, plus ouverte et plus adaptée à la nature imprévisible et changeable des métonymies, selon le type de problèmes qu'elles peuvent présenter pour le traducteur automatique. Par conséquent, en fonction de difficultés rencontrées dans un texte, nous voudrions proposer certaines solutions permettant de les surmonter, conformément à la méthode adoptée.

La méthodologie que nous avons appliquée à l'élaboration du classement mentionné est l'approche orientée objets de W. Banyśr. Proche de la méthode de G. Gross et partageant la même idée générale, selon laquelle « le sens d'un mot est fonction de son emploi et dépend, par conséquent des mots (attributs et opérateurs) qu'il accompagne » (A. C z e k aj, $2014: 242$ ), l'approche orientée objets s'en différencie par la manière de voir la description et la traduction des unités linguistiques. Or, à la différence de G. Gross, qui, dans la description des classes d'objets se limite à " fournir un listing explicite et exhaustif de tous les attributs et opérations possibles qui s’y appliquent» (A. Grigowic z, $2004: 23$;

${ }^{1}$ La méthode orientée objets s'insère dans le cadre théorique de la traduction «par règles de transfert ». Or depuis un certain temps la tendance en traduction automatique est de recourir à la statistique qui est parfois combinée avec les règles de transfert. 
cf. W. B a n y ś, 2002a, 2002b), W. Banyś ne s'arrête pas là et cherche à rendre compte des mécanismes généraux qui permettront à l'ordinateur de fournir la traduction correcte des mots dans tous les contextes dans lesquels ils peuvent apparaître. L'un de ces mécanismes, qui s'avère particulièrement efficace dans la désambiguïsation des sens des mots susceptibles de recevoir plusieurs interprétations différentes, est le cadre conceptuel (frame) considéré comme « ensemble de concepts typiquement liés, un prototype décrivant une situation » (A. Czekaj, 2014 : 242 ; cf. M. Minsky, 1975, 1985/1988 ; R. Schank, R. Abelson, 1977 ; B. Śmigielska, 2007, 2011, 2013). Autrement dit, le cadre constitue un contexte (proche et éloigné) dans lequel ces mots fonctionnent et qui devient particulièrement utile au moment où seuls les opérateurs et attributs ne sont pas suffisamment distinctifs pour la reconnaissance des emplois spécifiques. Ainsi, si l'ordinateur devrait traduire une expression appartenant à plusieurs classes d'objets distinctes et ayant, par conséquent plusieurs équivalents différents, il n'aurait qu'à chercher dans le texte certains indices, c'est-à-dire " les mots-clés qui le situerons dans le contexte convenable » (B. Ś mig i elsk a, 2007 : 255 ; cf. B. Śmigielska, 2011, 2013 ; A. C zekaj, 2011) et permettront de discerner le sens dont il s'agit. Le cadre est aussi primordial pour la compréhension des métonymies, et particulièrement des métonymies vives, produites spontanément pour les besoins d'une situation donnée, en tant que raccourcis langagiers, qui, sans recours au cadre semblent bizarres, incompréhensibles ou ridicules. En effet, les expressions du type, p. ex. :

(9) Cette table part demain.

(10) Le saxophone a la grippe aujourd'hui.

(11) Les nez longs gagnent.

isolées du contexte situationnel susciteraient certainement un sourire ou un étonnement chez les destinataires. Toutefois, placées dans un cadre concret, respectivement [restaurant d'hôtel] [orchestre] ou [photographie de portrait] et prononcées par, p. ex. un chef de restaurant (9), un chef d'orchestre (10) ou bien un membre de jury dans un concours de photographie de portrait (11), ces mêmes phrases deviennent tout à fait claires et compréhensibles. Certes, la compréhension est un facteur important pour le récepteur du message celui-ci étant un être humain, capable de déduire certaines informations du contexte. L'ordinateur a pourtant beau de recourir au cadre, étant donné que sa tâche n'est pas de comprendre mais de traduire l'expression donnée. En quoi donc le cadre faciliterait-il le processus de traduction automatique ? Or, la contribution du cadre à la traduction correcte est d'une valeur inestimable pour quelques raisons, que nous allons présenter dans ce qui suit.

Comme nous l'avons déjà signalé, ce qui définit l'objet (la classe d'objets) donné(e) permettant d'indiquer sa signification exacte et, par conséquent, sa tra- 
duction convenable dans la langue cible, ce sont les attributs et les opérateurs qui l'accompagnent. Naturellement, toutes ces caractéristiques ne sont propres à cette classe que dans les limites d'un cadre correspondant.

Par conséquent, l'objet p. ex. : table, qui pourrait être considéré comme, p. ex. "pièce de mobilier composée d'une surface plane horizontale disposée à hauteur convenable pour y prendre des repas et pour y déposer les objets et les mets nécessaires à ceux-ci ; en particulier, meuble construit spécifiquement à cet usage » (CNRTL, consulté le 25 avril 2020) ou bien comme "recueil de données (résultat de calculs, d'observations) groupées de façon systématique pour permettre une consultation rapide " (CNRTL, consulté le 25 avril 2020) serait caractérisé par les attributs et les opérateurs spécifiques résultant de l'appartenance de l'objet en question à l'un ou l'autre cadre conceptuel.

Ainsi, les attributs tels que, p. ex. :

grande, petite table

table carrée, ovale, rectangulaire, ronde

table de ferme, moderne, rustique, de style

table de bois, d'acajou, de chêne, de marbre, de sapin

table à abattants ; table pliante ; table de camping

table réservée,

etc.

et les opérateurs du type, p. ex. :

mettre, dresser la table

poser, servir qqc. sur la table

dîner à la table de qqn

s'installer à la table, s'asseoir devant, derrière une table

réserver la table

s'endormir à table, etc.

indiqueraient que l'objet en question est employé dans le premier sens et qu'il devrait être traduit en polonais par le substantif stót.

En revanche, les caractéristiques comme p. ex. :

table de multiplication

table d'addition

table de logarithmes

table de Mendeleïev

apprendre sa table de multiplication

étudier la table

mémoriser la table 
mettent en avant la deuxième signification de l'objet analysé et amènent l'ordinateur à choisir la traduction polonaise tablica, tabliczka.

Il se peut toutefois que la machine "tombe ", dans un texte à traduire, sur des opérateurs qui ne figurent pas dans la fiche descriptive de l'objet donné. Il en est ainsi avec l'expression citée plus haut Cette table part demain où l'objet table est accompagné du terme partir, cet opérateur n'étant certainement pas énuméré dans la description de l'objet mentionné. Dans cette situation, l'approche orientée objets propose de traduire l'expression donnée à la lettre, ce qui dans la plupart des cas, résultera d'une traduction correcte et compréhensible, au sein d'un cadre convenable, bien évidemment. Certes, la traduction ainsi obtenue ne sera peut-être pas toujours correcte et adéquate à cent pour cent, mais elle sera la plus probable, compte tenu d'une énorme fréquence et d'une grande variété de métonymies de ce type dans les langues naturelles, telles p. ex. :

- ceinture noire pour pratiquant de sports de combat du plus haut niveau C'est, en effet, à partir du moment qu'il est ceinture noire que le karatéka commence vraiment à intégrer le karaté à son mode de vie.

http://karatestroch.com/litterature/la-valeur-dune-ceinture/ (consulté le 15 février 2020)

- raquette pour joueur de tennis

Joueuse de tennis américaine Serena Williams, qui est la première raquette du monde, peut se marier avec interprète rap Drake, rapporte l'édition américaine Life \& Style.

http://fr.news-4-u.ru/les-journalistes-envoient-sereno-williams-marier.html (consulté le 15 février 2020)

- maillot jaune pour le meilleur cycliste

Ayant manqué quatre contrôles antidopage, il ne peut continuer à participer au Tour de France dont il est le maillot jaune.

http://pcmfrance.com/2005-tour-des-scandales/ (consulté le 16 février 2020)

- table pour clients d'hôtel occupant une table donnée pendant les repas

Cette table part demain.

- saxophone pour joueur de cet instrument

Le saxophone a la grippe aujourd'hui.

Dans tous les cas présentés ci-dessus, la traduction littérale serait correcte ne suscitant aucun étonnement non seulement chez les locuteurs natifs polonais mais aussi chez ceux parlant bien d'autres langues. Ainsi, le problème de la traduction de ce type de métonymies, qui dans la plupart des cas sont des métonymies vives, ne représente aucun problème pour la machine et n'exige pas, de ce fait, de classement particulier devant faciliter le choix du bon équivalent de l'expression donnée dans la langue cible. En effet, la plupart de ces expressions métonymiques, pourraient être traduites au pied de la lettre, même sans avoir besoin de faire appel au cadre. 
Le recours au cadre devient pourtant très important lorsque l'expression donnée pourrait être interprétée et, par conséquent traduite, de plusieurs façons différentes. À cet égard, on pourrait se servir du lexème p. ex. : parfum, qui peut engendrer trois significations différentes devant être traduit à l'aide d'au moins trois substantifs polonais, à savoir : zapach, perfumy et smak. Puisque dans l'approche orientée objets, différentes significations des unités lexicales sont séparées généralement grâce aux attributs et opérateurs qui constituent les premiers critères de la désambiguïsation du sens des mots, il suffit de regarder quels opérateurs qui accompagnent le substantif parfum entraineraient la traduction zapach et lesquels feraient choisir l'équivalent perfumy (A. C z ek aj, 2017).

Ainsi, le sens du substantif parfum considéré comme " odeur agréable et pénétrante d'origine naturelle ou artificielle » (CNRTL, consulté le 2 mai 2020), dont les extensions métaphoriques peuvent concerner :

- " ce qui laisse un souvenir agréable ; rappel d'une évocation touchante »,

- « atmosphère délicate qui entoure un être ou une chose » (CNRTL, consulté le 2 mai 2020),

et par conséquent, la traduction polonaise zapach seraient évoqués par les attributs et les opérateurs tels que, p. ex. :

\begin{tabular}{|c|c|c|}
\hline parfum doux & - & przyjemny zapach \\
\hline parfum entêtant & - & odurzający zapach \\
\hline parfum discret & 一 & dyskretny zapach \\
\hline parfum puissant & 一 & mocny zapach \\
\hline parfum sensuel & - & zmystowy zapach \\
\hline parfum frais & - & świeży zapach \\
\hline parfum lourd & - & ciężki zapach \\
\hline parfum fort & - & ostry zapach \\
\hline parfum tenace & - & trwaty zapach \\
\hline parfum de la rose & - & zapach róży \\
\hline parfum du pain & - & zapach chleba \\
\hline parfum du succès & - & zapach sukcesu \\
\hline parfum de l'enfance & - & zapach dzieciństwa \\
\hline parfum entête & - & zapach odurza \\
\hline parfum se répand & - & zapach unosi się \\
\hline dégager un parfum & - & wydzielać zapach \\
\hline humer un parfum & 一 & wdychać zapach \\
\hline composer un parfum & - & komponować zapach \\
\hline
\end{tabular}

Quant à la signification «substance aromatique agréable à l'odorat, liquide ou solide, d'origine naturelle ou artificielle » (CNRTL, consulté le 2 mai 2020) exigeant l'équivalent polonais perfumy, elle serait suggérée à l'ordinateur par la présence des opérateurs du type, p. ex. : 


$\begin{array}{lll}\text { parfums pour femmes } & - & \text { perfumy dla kobiet } \\ \text { parfums pour hommes } & - & \text { perfumy dla mężczyzn } \\ \text { parfums de femme } & - & \text { perfumy damskie } \\ \text { parfums d'homme } & - & \text { perfumy męskie } \\ \text { parfum cher } & - & \text { drogie perfumy } \\ \text { parfum en vaporisateur } & - & \text { perfumy w atomizerze } \\ \text { parfums de marque } & - & \text { markowe perfumy } \\ \text { parfum sent bon / mauvais } & - & \text { perfumy pachna ładnie / brzydko } \\ \text { acheter un parfum } & - & \text { kupić perfumy } \\ \text { vendre des parfums } & - & \text { sprzedawać perfumy } \\ \text { s'asperger de parfum } & - & \text { skropić się perfumami } \\ \text { se mettre du parfum } & - & \text { perfumować się }\end{array}$

Divers textes linguistiques témoignent pourtant que, malgré la présence des attributs et opérateurs cités ci-dessus, qui devraient indiquer le choix soit de l'un soit de l'autre équivalent polonais, les substantifs qui apparaissent dans les traductions polonaises sont appliqués "à l'envers ». Cette possibilité de remplacement pourrait s'expliquer d'un côté par le même cadre d'emploi, qui est la [cosmétique] dans les deux cas, et de l'autre - par l'utilisation fréquente des métonymies, donc des raccourcis de pensée, qui font lier « la substance aromatique agréable à l'odorat » à « l'odeur agréable et pénétrante ». Ainsi, la distinction de ces deux emplois du mot analysé paraît toute simple, étant donné que l'ordinateur peut choisir n'importe lequel des deux équivalents polonais proposés et la traduction restera correcte. Cette inversion des équivalents zapach ou perfumy n'est pourtant pas possible dans tous les cas. En effet, il existe des opérateurs qui dirigent la machine plutôt vers l'un que vers l'autre des équivalents polonais, sans qu'on puisse les interchanger car le remplacement entraînerait la traduction inadéquate. Il en va ainsi avec des expressions, p. ex. :

$\begin{array}{lll}\text { flacon de parfum } & - & \text { flakonik perfum } \\ \text { s'inonder de parfum } & - & \text { oblać się perfumami } \\ \text { s'asperger de parfum } & - & \text { skropić się perfumami } \\ \text { parfum du printemps } & - & \text { zapach wiosny } \\ \text { parfum de liberté } & - & \text { zapach wolności } \\ \text { parfum du succès } & - & \text { zapach sukcesu } \\ \text { parfum de la rose } & - & \text { zapach róży } \\ \text { parfum du pain } & - & \text { zapach chleba } \\ \text { parfum de chocolat } & - & \text { zapach czekolady } \\ \text { parfum du musc } & - & \text { zapach piżma } \\ \text { parfum de bois } & - & \text { zapach drewna } \\ \text { parfum de l'argent } & - & \text { zapach pieniędzy }\end{array}$


Les exemples cités ci-dessus font voir que l'appartenance du substantif parfum à une classe d'objets déterminée permet d'indiquer sa traduction soit à l'aide de l'équivalent zapach soit au moyen de l'équivalent perfumy. Si notamment l'objet en question fait partie de l'une des classes, que l'on pourrait nommer comme p. ex. : [saisons et périodes de l'année] : zapach wiosny, zapach wakacji, [produits alimentaires] : zapach chleba, zapach czekolady, [plantes] : zapach róży, zapach drzew, zapach ziót, [matières animales ou végétales] : zapach drewna, zapach piżma ou enfin [noms abstraits désignant des états et qualités désirés] : zapach sukcesu, zapach wolności, zapach pieniędzy, la machine serait dirigée vers le choix du substantif polonais zapach. Par contre, l'appartenance du mot analysé à la classe des [liquides] entraînerait la traduction perfumy (cf. A. C z e k a j, 2017).

Par conséquent, lorsque le contexte immédiat du substantif parfum serait composé des attributs ou opérateurs mentionnés, qui acceptent seulement une possibilité de traduction, le traducteur automatique n'aurait qu'un seul équivalent polonais disponible dans la base des données. Dans les autres cas, il pourrait adopter les deux possibilités vu qu'en général, les deux auraient pour effet la traduction acceptable.

Nous avons toutefois signalé, dans ce qui précède que le substantif analysé possède trois significations, dont également « substance aromatique agréable au goût que l'on incorpore aux boissons, aux mets, aux desserts » (CNRTL, consulté le 2 mai 2020) ou, par métonymie, le goût lui-même. Cet emploi métonymique, qui exige le choix d'un autre équivalent polonais - smak, vient compliquer un peu la traduction automatique.

Certes, il est évident que la traduction polonaise smak peut résulter de l'emploi du substantif parfum dans un cadre gastronomique et, le cas échéant, fait référence à différents produits alimentaires tels : glaces parfum vanille, yaourts parfum fraise, gâteaux parfum chocolat, etc. Mais il est évident aussi que les mêmes parfums peuvent accompagner différents produits de beauté qui, en tant qu'éléments provenant du cadre cosmétique, exigent l'équivalent polonais zapach, p. ex. : zapach wanilii (waniliowy), zapach czekolady (czekoladowy).

Pour cette raison, le recours au cadre conceptuel devient indispensable afin d'aider la machine à découvrir dans quel sens le mot analysé est employé et à choisir l'équivalent adéquat dans la langue cible.

Ainsi, si dans l'environnement (non seulement immédiat) de parfum l'ordinateur trouve des mots relatifs à la [cosmétique], il serait orienté vers le choix de l'équivalent zapach, tandis que l'accumulation des termes se rapportant à la [gastronomie/cuisine] favoriserait le choix de l'équivalent smak.

Pour illustrer cette constatation, regardons quelques exemples de textes :

(1) Pour 12 yaourts dans la MultiDélice: 1 L200 de lait entier, 1 yaourt nature, 250g de crème glacée

Pour une yaourtière 7 ou 8 pots : 1 L de lait, 1 yaourt, $200 \mathrm{~g}$ de crème glacée 
Faire fondre la crème glacée et laisser refroidir.

Mélanger la crème glacée avec le yaourt et le lait en mélangeant bien.

Verser la préparation dans les pots et mettre en yaourtière $8 \mathrm{~h}$. A la fin du programme, mettre au frais au moins 4 h.

Les morceaux de biscuits remontent du coup on obtient un yaourt multicouches: couche de yaourt biscuitée puis couche yaourt parfum vanille et au fond une mini couche yaourt chocolat!

http://lacuisinededey.blogspot.com/2013/08/yaourts-facon-profiteroles.html (consulté le 30 mai 2020)

(2) Crêpes parfum cannelle

Chacun a son avis sur le parfum de la pâte à crêpes, rhum, fleur d'oranger, mais rares sont ceux qui testent avec de la cannelle dans la pâte! J'ai trouvé cette idée dans une fiche Elle de la bloggeuse Marjolaine Daguerre, et j'ai adoré ! d'autant qu'elle fait cuire les crêpes dans du beurre salé ;) Une heure de repos pour la pâte suffit. Il faut un feu vif pour la cuisson des crêpes et une poèle bien chaude.

http://melusine064.canalblog.com/archives/2019/02/02/37060551.html

(consulté le 30 mai 2020)

(3) Je pris mes vêtements puis allai dans ma salle de bains, elle était blanche avec quelques carreaux rouges et le carrelage blanc avec un tapis de bain rouge. Il y avait une petite douche à l'italienne dans le coin de la pièce. Je posai mes affaires sur le meuble de la salle de bains, puis me déshabillai, j'entrai dans la douche puis fermai le petit rideau noir. Je me savonnai avec mon gel douche parfum chocolat, puis me lavai les cheveux avec du shampoing aux fleurs.

https://real-fiction-cinderella.skyrock.com/ (consulté le 30 mai 2020)

(4) Savon parfum framboise à l'huile d'argan bio

La senteur fruitée et sucrée de framboise vous procurera une sensation de fraîcheur immédiate. Pour la toilette quotidienne ou pour offrir, cette savonnette pur végétal est enrichie à l'huile d'argan bio, riche en acides gras essentiels comme l'acide linoleique, en oméga-6, anti-oxydants qui préviennent le dessèchement de la peau. Convient pour tous types de peau.

https://www.olive-oil-only.fr/vente+savon-parfum-framboise-a-l-huile-dargan-bio+275.html (consulté le 30 mai 2020)

À travers les exemples présentés, on voit que les indices qui se trouvent dans les textes (1) et (2) comme p. ex. : yaourt, lait entier, crème glacée, yaourtière, faire fondre, crème glacée, laisser refroidir, biscuits, pâte à crêpes, cannelle, beurre salé, poêle bien chaude concernent le domaine [gastronomie, cuisine], ce qui ferait orienter la machine vers le choix du substantif polonais smak. En revanche, les mots-clés qui apparaissent dans les fragments (3) et (4) tels que, p. ex. : salle de bains, douche, je me savonnai, gel douche, me lavai les cheveux, shampoing, savon, sensation de fraîcheur, toilette quotidienne, savonnette, dessèchement de la peau, se rapportent au cadre [cosmétique, soins du corps] grâce à quoi l'ordinateur choisirait l'équivalent polonais zapach. 
Ainsi, dans le but de résoudre le problème de la traduction des expressions polysémiques (dont également métonymiques), la description détaillée du cadre conceptuel, riche en points de repère spécifiques, c'est-à-dire en expressions typiques pour une situation concrète, devient une chose primordiale pour que l'ordinateur puisse trouver la traduction convenable, étant donné que " plus il y a de détails caractéristiques pour un cadre donné plus la chance de diriger la machine vers le bon choix de l'équivalent est grande » (A. Czek aj, 2017 : 50).

Un autre problème lié à la traduction automatique des métonymies concerne les expressions marquées des influences culturelles, historiques, politiques, sociologiques ou religieuses, donc propres pour une société donnée. Très souvent, ces métonymies sont ancrées dans la tradition, dans le système de croyances, qui est la base de la vision du monde de cette société et qui façonne ses pensées, ses actions et sa perception. Par conséquent, vu leur caractère spécifique, ces métonymies sont compréhensibles généralement juste pour la communauté qui les a construites et de ce fait, sont difficilement traduisibles, en tout cas de façon automatique.

Dans la plupart des cas, les expressions de ce type ne peuvent pas être traduites de façon littérale, parce que les constructions obtenues de cette manière ne seront généralement pas claires pour les utilisateurs d'autres langues. Parmi les métonymies en question, on pourrait trouver des exemples polonais comme p. ex. : biate kotnierzyki, moherowe berety, białe fartuchy ou français, tels que : cols bleus, robes noires, gilets rouges, gilets jaunes et beaucoup d'autres.

De telles constructions métonymiques sont très souvent utilisées par les journalistes, qui veulent éviter les redondances et donner du rythme à leurs textes, ce qui ne va malheureusement pas de pair avec la facilité de traduction automatique. Bien au contraire, la traduction littérale de telles constructions les rend peu intelligibles pour les utilisateurs de la langue cible.

Ainsi, les phrases comme, p. ex. :

Białe fartuchy protestuja.

http://www.archiwum.wyborcza.pl/Archiwum/1,0,217738,19960617RPDGW,Bia

le_fartuchy_protestuja,.html (consulté le 30 mai 2020)

Pour finir, les gilets rouges n'avaient absolument aucune information cohérente à communiquer.

https://malignec.transilien.com/2014/07/17/programme-de-circulation-des-trainspour-ce-jeudi-17-et-pour-vendredi-18-juillet/

(consulté le 20 octobre 2016)

I pokazać, że my nie jesteśmy moherowe berety.

https://www.gdansk.pl/wiadomosci/gdanscy-seniorzy-maja-swoja-olimpiade-jako-pierwsi-wpolsce-beda-mieli-tez-wszechnice, a, 87565

(consulté le 20 octobre 2016) 
traduites mot à mot, ne diraient rien aux destinataires français et polonais car même si les traductions obtenues de cette façon seraient correctes du point de vue syntaxique, leur sens resterait indéchiffrable pour les récepteurs des deux langues concernées. En effet, l'expression tabliers blancs (pour białe fartuchy) renverrait un Francophone aux vêtements de protection, portés habituellement par les cuisiniers, alors que béret de mohair (pour moherowy beret) ferait référence à une toque en laine angora pour femmes. De même, l'expression czerwone kamizelki (pour gilets rouges) indiquerait à tout Polonais un type de vêtement qui couvre le corps, généralement sans manches et de couleur rouge.

Par conséquent, pour les constructions métonymiques de ce type où la traduction littérale n'aboutit pas aux résultats satisfaisants, générant des phrases incompréhensibles pour le récepteur, la méthode orientée objets a prévu une rubrique spéciale dans la fiche descriptive des unités lexicales. Il s'agit de la catégorie des extensions où l'on place toutes les expressions dont la signification passe les limites du cadre admis, ce qui rend impossible leur traduction textuelle à cause de l'opacité de la version ainsi obtenue. Ainsi, toutes les expressions pareilles (n'ayant généralement pas de correspondant fidèle dans la langue cible) seraient accompagnées de tels équivalents (métonymiques ou non) qui transmettraient, de façon la plus concise possible, le sens qu'elles communiquent, comme p. ex. kolejowe stuzby informacyjne pour gilets rouges, catholiques traditionnalistes pour moherowe berety ou infirmiers ou blouses blanches pour biate fartuchy (cf. A. Czekaj, 2015).

La solution proposée ne s'applique bien sûr qu'à ces expressions dont la traduction littérale n'a aucune raison d'être dans la langue cible car il existe des métonymies qui, traduites au pied de la lettre, véhiculent le même sens dans l'autre langue. La correspondance de ce type s'observe dans les expressions comme, p. ex. : białe kolnierzyki, niebieskie kolnierzyki ou żólte kamizelki qui renvoient aux mêmes groupes sociaux, respectivement, des travailleurs de bureau (intellectuels), des travailleurs physiques et des membres du mouvement s'opposant à l'augmentation des taxes sur les carburants, et plus globalement à une baisse du pouvoir d'achat.

Il existent cependant des métonymies dont la traduction textuelle existe dans la langue cible mais produit des connotations différentes. Il en est ainsi avec l'expression p. ex. : robes noires, qui, traduite en polonais comme czarne sukienki fait référence aux représentants du clergé, et notamment aux prêtres, tandis que pour les locuteurs francophones, l'expression robes noires peut renvoyer également aux personnes exerçant la profession d'avocat. Dans ce cas-là, c'est une fois de plus que le recours au cadre s'avère nécessaire pour diriger l'ordinateur soit vers la traduction littérale soit vers celle par paraphrase, énumérée parmi les extensions de l'objet robe considéré comme " vêtement long et à manches porté comme signe d'appartenance à un état, à une fonction ou à une profession inspirant l'autorité et le respect » (CNRTL, consulté le 30 mai 2020). 
Par conséquent, si l'ordinateur trouve dans l'environnement de l'expression robes noires des mots relatifs à [religion], il pourra traduire cette expression au pied de la lettre. Si par contre le voisinage de l'expression en question constituent des termes concernant le cadre [juridiction], il devra choisir l'équivalent de l'expression en question, listé dans la fiche descriptive comme przedstawiciele stanu adwokackiego. On pourrait le faire observer à l'aide des textes suivants :

Comme d'autres populations nomades du Subarctique canadien, les Atikamekw auraient tous affiché, du moins à la lumière des données historiques disponibles, une même attitude collective à l'égard des robes noires et de leur message religieux. Ainsi, par exemple, au temps de la mission catholique, soit durant quelques semaines chaque été, la grande majorité des Atikamekw participait avec enthousiasme aux diverses activités organisées par les missionnaires : messes, prières, processions. Or, ce portrait d'Atikamekw essentiellement tous convertis et fervents pratiquants, véhiculé surtout par les écrits des missionnaires, s'accorde plutôt mal avec, d'une part, le fait que la conversion constituait une démarche avant tout individuelle et que, par conséquent, tous les autochtones d'un même groupe n'adhéraient pas nécessairement de la même façon et avec la même intensité à une nouvelle religion.

http://schec.cieq.ca/documents_pdf/revue_2003.pdf(consulté le 2 mai 2020)

Grève des avocats : les robes noires distribuent des roses à Clermont-Ferrand

Les avocats clermontois ont mené une nouvelle action dans le cadre de leur mouvement contre la réforme des retraites. Ils ont fabriqué puis distribué ce mardi aprèsmidi de fausses roses, rouges et noires, aux magistrats du tribunal judiciaire.

Ils ne manquent pas d'imagination. Dans leurs plaidoiries mais aussi dans leurs actions contre la réforme des retraites.

https://www.francebleu.fr/infos/faits-divers-justice/greve-des-avocats-les-robes-noiresdistribuent-des-roses-a-clermont-ferrand-1582042142

(consulté le 2 mai 2020)

où les indices : religieux, mission catholique, missionnaires, messes, prières, processions, convertis, religion entraîneraient la traduction littérale et ceux du type : avocats, magistrats, tribunal judiciaire, plaidoiries feraient choisir à la machine l'expression polonaise mentionnée.

Le dernier type de métonymies qui, à notre avis, pourrait paraître problématique pour le traducteur automatique sont les expressions, souvent basées sur les relations métonymiques du type [inventeur pour invention], [producteur pour produit] ou [lieu pour institution] qui trouvent leur origine dans les noms propres. Au sein de cette catégorie, il y a des termes, qui se sont tellement enracinés dans la langue qu'ils ne sont plus considérés comme métonymies, comme expressions « bizarres » exigeant le recours au cadre pour être comprises, mais comme les noms communs. Parmi ces expressions on peut trouver des mots comme, p. ex. : (1) diesel, (2) jacuzzi ou polonais (3) rower, (4) prysznic, qui à présent, désignent différents objets, respectivement : (1) véhicule équipé du moteur diesel, le moteur 
lui-même ou le carburant pour ce type de moteur, (2) petit bassin pourvu de jets d'eau sous pression qui créent des remous relaxants, (3) bicyclette ou (4) installation où l'eau jaillit en coulant à travers un tamis spécial ou au fait de se laver à l'aide de cette installation, et ne font pas penser aux noms de leurs créateurs ou fabricants. Par conséquent, bien que les mots en question soient universellement connus, on ne se souvient presque plus de quoi ils ont été motivés.

Certainement on se pose ici la question de savoir pour quelle raison les expressions de ce type pourraient être considérées comme problématiques pour l'ordinateur, si elles constituent des mots simples, donc sans doute présents dans la base des données de l'ordinateur avec un équivalent correspondant. Pourtant, la chose n'est pas si évidente dans tous les cas car dans cette même catégorie, il y a aussi des expressions qui sont entrées dans le langage courant et dont le caractère métonymique n'est pas ressenti non plus par les locuteurs mais qui ne sont pas tellement connues comme celles présentées plus haut.

À titre d'exemple, on pourrait énumérer les mots tels que, p. ex. : cantal, kleenex, sopalin, ou bien laguiole. Or, les utilisateurs d'autres langues peuvent ne pas savoir que les substantifs français mentionnés se réfèrent à : type de fromage, mouchoirs jetables, essuie-tout en papier et couteaux, vu qu'ils ont été construits à la base des noms des lieux de la fabrication des objets en question (cantal, laguiole) ou de la marque de leur fabricant (kleenex, sopalin). Leur traduction automatique ne constitue effectivement aucun problème pour l'ordinateur qui n'a qu'à les laisser inchangés, c'est-à-dire en version originale en tant qu'éponymes. Une difficulté éventuelle concernerait plutôt la compréhension des termes en question, celle-ci étant pourtant le résultat de connaissance insuffisante de la langue source chez les récepteurs.

La connaissance insuffisante du français n'est cependant pas la seule source de l'incompréhension, celle-ci pouvant résulter également d'un faible savoir général sur le monde.

En effet, il existe nombre d'expressions métonymiques faisant référence à, par exemple, différentes institutions au moyen du nom de l'endroit où elles se trouvent. Le caractère métonymique de ces expressions est plus perceptible parait-il, cependant leur compréhension dépend fortement du degré de leur enracinement dans une langue donnée. Si donc, on peut facilement identifier les référents désignés au moyen des termes tels que, p. ex. : Maison Blanche ou Wall Street, sachant qu'il s'agit du président des États-Unis de l'Amérique dans le premier cas et de la plus grande bourse mondiale - dans l'autre, il y a des métonymies qui ne disent pas grand-chose aux destinataires. Il y est question des expressions qui indiquent, par métonymie, des endroits ou personnes importants du point de vue du fonctionnement d'une société donnée mais dont l'importance n'est peut-être pas la même pour une autre société. Tel est le cas des noms, p. ex. : le Quai d'Orsay, qui est la métonymie utilisée pour désigner le ministère des Affaires étrangères du gouvernement français, Bercy - pour le ministère de l'Économie et des Finances, la 
rue de Valois - pour le ministère de la Culture et de la Communication, la place Beauvau — pour le ministère de l'Intérieur ou enfin Matignon — pour le siège du gouvernement français. Bien sûr, ce qui témoigne l'emploi métonymique des noms en question, ce sont les opérateurs qui les accompagnent et le cadre correspondant, indiquant soit les représentants du ministère donné, p. ex. :

Le Quai d'Orsay prend l'initiative de coordonner ces mouvements et crée, en janvier 1916, la Maison de la Presse.

https://www.persee.fr/doc/efr_0000-0000_1984_act_54_2_2301 (consulté le 2 mai 2020)

soit un endroit de Paris.

Le quai d'Orsay est un quai situé sur la rive gauche de la Seine dans le $7^{e}$ arrondissement de Paris.

https://www.aucoeurdelimmo.com/actualites/le-quai-d-orsay-en-tete-du-top-100-desrues-les-plus-cheres-de-paris_1712.html (consulté le 2 mai 2020)

Et voilà de nouveau que le traducteur automatique ne devra pas traduire les noms propres mentionnés, qui, en tant que tels, n'exigent pas d'être traduits, ce qui les rendra peut-être incompréhensibles pour certains, ne connaissant pas les noms ni la localisation des institutions concernées. Par conséquent, les problèmes générés par les métonymies de ce type, ne se situerait pas du côté du traducteur automatique mais plutôt du côté du récepteur, qui, faute du savoir suffisant, pourrait considérer la traduction obtenue comme mal effectuée et fautive.

En conclusion, étant donné tout ce que nous avons dit jusqu'ici et tenant compte d'une très grande fréquence et diversité de constructions métonymiques dans les textes, nous tenons à souligner qu'une classification rigoureuse et pour cela inflexible des métonymies ne présente aucune utilité pratique du point de vue de la traduction automatique. Par conséquent, le seul classement qui nous paraît justifié et probant est celui qui permettrait de rendre compte du procédé au moyen duquel une métonymie donnée pourrait être traduite de façon automatique. $\mathrm{Ce}$ classement prend en considération :

1. métonymie traduite à l'aide d'une métonymie - le plus souvent de façon littérale (cette table part demain — ten stolik jutro wyjeżḋa) ou avec le recours au cadre (quel parfum vous préférez ? - jaki zapach, smak, perfumy pan(i) preferuje?) ;

2. métonymie métaphorique traduite à l'aide d'une métonymie métaphorique (cols blancs - biate kotnierzyki) ou par paraphrase (moherowe berety - catholiques traditionnalistes) ;

3. métonymie qui n'est plus ressentie comme telle par les locuteurs — traduite selon la connaissance de la langue (dans le cas des expressions qui sont entrées dans le lexique d'une langue : prysznic - douche) ou non traduite (cantal, 
Bercy), comprise par le récepteur grâce à sa connaissance de la langue ou son savoir général.

En bref, la métonymie ne représente pas un grand problème pour l'ordinateur vu que la majorité des expressions métonymiques peuvent être traduites, d'abord au moyen des opérateurs qui se manifestent dans le contexte de leur emploi et qui constituent le tout premier critère du choix de l'équivalent adéquat; ensuite, avec le recours au cadre conceptuel, dont les indices servent d'outils complémentaires pour sélectionner le correspondant convenable ; et finalement grâce à l'absence de classements rigides, ce qui permet la traduction littérale. Il faudrait remarquer tout de même que la traduction effectuée sera toujours une traduction par préférence, donc la plus probable dans une situation donnée, ce qui ne s'avérera peutêtre pas correct dans tous les cas. Nous trouvons cependant qu'une telle solution du problème de la traduction des métonymies serait beaucoup plus efficace par rapport aux approches basées sur des classifications détaillées, qui « interdisent » l'emploi de métonymie, là où il peut bel et bien avoir lieu.

\section{Références citées}

A r n a u d P.J., 2009 : Détecter, classer et traduire les métonymies (anglais et français). Passeur de mots, passeurs d'espoir. Lexicologie, terminologie et traduction face au défi de la diversité : Actes des huitièmes journées scientifiques du Réseau de chercheurs LTT. Lisbonne : Agence Universitaire de la Francophonie, 503-516.

B a n y ś W., 2002a : « Bases de données lexicales électroniques — une approche orientée objets. Partie I : Ouestions de modularité ». Neophilologica 15, 7-28.

B a n y ś W., 2002b : « Bases de données lexicales électroniques — une approche orientée objets. Partie II : Questions de description ». Neophilologica 15, 206-248.

Blank A., 1999 : "Co-presence and succession: A cognitive typology of metonymy". In: K.-U. P a n th e r, G. R a d d e n, dir.: Metonymy in Language and Thought. Amsterdam, Benjamins, 169-191.

Czekaj A., 2011 : «Question de métonymie dans la traduction automatique ». Neophilologica 23, 136-149.

Czekaj A., 2014 : «Comment comprendre la classe d'objets? ». Neophilologica 26, $232-244$.

Czekaj A., 2015 : «Cette table part demain — la faute du traducteur ou l'intention de l'auteur? - à propos de la métonymie dans la traduction automatique ». Neophilologica $27,33-44$.

Czekaj A., 2017 : «Quelques remarques pour vous mettre au parfum à propos du parfum ». Neophilologica 29, 40-54.

C z e k aj A., 2018 : « Perception et métonymie — problèmes de traduction automatique ». Neophilologica $30,76-88$. 
C z e k aj A., 2019 : « Classement de métonymies et son utilité dans la traduction automatique ». Neophilologica 31, 106-124.

Grigowicz A., 2004 : « Parties du corps et approche orientée objets ». Neophilologica $16,20-41$.

Grigowicz A., 2007 : « Parties du corps et leurs opérateurs dans l'approche orientée objets ». Neophilologica 19, 228-242.

Gros s G., 1994a : "Classes d'objets et description des verbes ». Langages 115, 15-30.

Gro s s G., 1994b : «Classes d'objets et synonymie ». Annales Littéraires de l'Université de Besançon, Série Linguistique et Sémiotique 23, 93-102.

Gros s G., 1995 : « Une sémantique nouvelle pour la traduction automatique : les classes d'objets ». In : La Tribune des industries de la langue et de l'information électronique, $17-19$.

Gros s G., 2008 : «Les classes d'objets ». Lalies 28, 111-165.

He n ry A., 1971 : Métonymie et métaphore. Paris, Klincksieck.

Kövecses Z., Radden G., 1998 : "Metonymy: Developing a cognitive linguistic view". Cognitive Linguistics, 9, 37-77.

Lakoff G., Johnson M., 1980 : Metaphors We Live By. Chicago, University of Chicago Press.

Lak off G., John s o n M., 1988 : Metafory w naszym życiu. Warszawa, PIW.

Le colle M., 2001 : "Métonymie dans la presse écrite : entre discours et la langue ». Travaux neuchâtelois de linguistique 34/35, 153-170.

M a s s o u s s i T., 2007: «Métonymie et classes d'arguments». Neophilologica 19, 119—137.

Mas s o us s i T., 2008 : Mécanisme de la métonymie : approche syntactico-sémantique. Thèse de doctorat. Université de Paris 13, Villetaneuse.

Ma s s o u s s i T., 2009 : « Transfert sémantique et classes d'objets ». Contemporary Linguistics 67, 45-68.

M insky M., 1975 : A Framework for Representing Knowledge. In: P.H. W in st on, ed.: The psychology of computer vision. New York, McGraw-Hill Book.

Min s k y M., 1985 : The Society of Mind. New York, Simon and Schuster.

M in sk y M., 1988 : La société de l'esprit. Trad. J. He n ry. Paris, InterÉditions.

Mori e r H., 1961 [1989] : Dictionnaire de poétique et de rhétorique. Paris, PUF.

P eirsman Y., Ge era erts D., 2006 : "Metonymy as a prototypical category". Cognitive Linguistics 17, 269-316.

S ch a n k R., A b e ls on R., 1977 : Scripts, Plans, Goals and Understanding, An inquiry into human knowledge structure. Hillsdale, NJ: Lawrence Erlbaum Associates.

S t a 11 a rd D., 1993 : "Two kinds of metonymy". In: Proceedings of the 31st annual meeting on Association for Computational Linguistics, 87-94.

Śmigielska B., 2007 : «Remarques sur la traduction automatique et le contexte ». Neophilologica 19, 253-267.

Śmigi elska B., 2011 : " Rôle et description du contexte dans la traduction automatique des textes — approche orientée objets ». Romanica Cracoviensia 11, 422-432.

Ś m i g i els k a B., 2013 : « Description des cadres dans l'approche orientée objets en vue de la traduction assistée par ordinateur ». Roczniki Humanistyczne 61 (8) : Lingwistyka korpusowa i translatoryka, 49-60. 